\title{
Ruthenium-Catalyzed Anti-Markovnikov Hydroamination of Vinylarenes
}

\author{
Supporting Information
}

Masaru Utsunomiya and John F Hartwig*

Yale University, Department of Chemistry, P.O. Box 208107, New Haven, CT 06520-8107 
General comments. All reactions were performed under a nitrogen atmosphere using standard Schlenk and drybox techniques. Vinylarenes, amines, ligands, ruthenium catalyst precursors except $\mathrm{Ru}\left(2,3\right.$-dimethyl-1, 3-butadiene)(acac) ${ }_{2}$, 1, 4-dioxane anhydrous, triflic acid, and trifluoroacetic acid were purchased from commercial sources and were used without further purification. $\mathrm{Ru}\left(2,3\right.$-dimethyl-1, 3-butadiene)(acac) ${ }_{2}$ was synthesized by literature procedures. ${ }^{1}$ NMR spectra were recorded on Bruker DPX 400 and $500 \mathrm{MHz}$ instruments. Elemental analyses were performed by Robertson Microlit Laboratories, Madison, NJ.

\section{General Procedure for the Ruthenium-Catalyzed Hydroamination of Vinylarenes}

(Table 2). In a drybox, $\mathrm{Ru}(\operatorname{cod})(2 \text {-methylallyl) })_{2}(16.0 \mathrm{mg}, 50 \square \mathrm{mol})$ and 1,5bis(diphenylphosphino)pentane $(30.8 \mathrm{mg}, 70 \square \mathrm{mol})$ were suspended in $0.20 \mathrm{~mL}$ of dioxane in a screw-capped vial. Vinylarenes $(2.00 \mathrm{mmol})$ and amines $(1.00 \mathrm{mmol})$ were placed into a second small vial and dissolved in $0.30 \mathrm{~mL}$ of 1,4-dioxane. The vials were sealed with a cap containing a PTFE septum and removed from the drybox. Triflic acid $(8.8 \square 1,0.10 \mathrm{mmol})$ was added to the catalyst suspension. The solution of vinylarenes and amines was then added to the catalyst suspension. The reaction mixture was stirred at $100{ }^{\circ} \mathrm{C}$ for $24 \mathrm{~h}$. After heating, the reaction mixture was directly adsorbed onto silica gel and purified by flash column chromatography.

$N$-(2-Phenethyl)morpholine (Table 2, Entry 1). ${ }^{2}$ The general procedure was followed with styrene (229 $\square 1,2.00 \mathrm{mmol})$ and morpholine $(87.2 \mathrm{mg}, 1.00 \mathrm{mmol})$. The reaction mixture was purified by flash column chromatography (20\% EtOAc in hexane) to give $183 \mathrm{mg}(96 \%)$ of the hydroamination product: ${ }^{1} \mathrm{H}$ NMR (400 MHz, $\left.\mathrm{CDCl}_{3}, \mathrm{TMS}\right) \square 2.44(\mathrm{t}, J=4.6 \mathrm{~Hz}, 4 \mathrm{H}), 2.55$ $2.62(\mathrm{~m}, 2 \mathrm{H}), 2.77-2.83(\mathrm{~m}, 2 \mathrm{H}), 3.74(\mathrm{t}, J=4.6 \mathrm{~Hz}, 4 \mathrm{H}), 7.17-7.23(\mathrm{~m}, 3 \mathrm{H}), 7.25-7.31(\mathrm{~m}, 2 \mathrm{H})$; ${ }^{13} \mathrm{C}\left\{{ }^{1} \mathrm{H}\right\} \mathrm{NMR}\left(100 \mathrm{MHz}, \mathrm{CDCl}_{3}\right) \square 33.3,53.6,60.8,66.9,126.0,128.3,128.6,140.0$.

$N$-(2-Phenethyl)piperidine (Table 2, Entry 2). ${ }^{2}$ The general procedure was followed with styrene $(229 \square 1,2.00 \mathrm{mmol})$ and piperidine $(85.0 \mathrm{mg}, 1.00 \mathrm{mmol})$. The reaction mixture was purified by flash column chromatography (20\% EtOAc in hexane) to give $173 \mathrm{mg}(91 \%)$ of the hydroamination product: ${ }^{1} \mathrm{H}$ NMR (400 $\mathrm{MHz} \mathrm{CDCl}_{3}$, TMS) $\square$ 1.40-1.49 (m, 2H), 1.57-1.66 (m, 4H), 2.34-2.56 (m, 4H), 2.51-2.55 (m, 2H), 2.77-2.83 (m, 2H), 7.14-7.22 (m, 3H), 7.24-7.31 $(\mathrm{m}, 2 \mathrm{H}) ;{ }^{13} \mathrm{C}\left\{{ }^{1} \mathrm{H}\right\}$ NMR $\left(100 \mathrm{MHz}, \mathrm{CDCl}_{3}\right) \square 24.4,25.9,33.5,54.5,61.4,125.9,128.3,128.7$, 140.6.

1-Phenyl-4-(2-phenylethyl)piperazine (Table 2, Entry 3). ${ }^{3}$ The general procedure was followed with styrene (458 $\square 1,4.00 \mathrm{mmol})$ and 4-phenylpiperazine (162 mg, $1.00 \mathrm{mmol})$. The 
reaction mixture was purified by flash column chromatography (20\% EtOAc in hexane) to give $171 \mathrm{mg}(64 \%)$ of the hydroamination product: ${ }^{1} \mathrm{H}$ NMR (400 MHz, $\mathrm{CDCl}_{3}$, TMS) $\square 2.61-2.68$ $(\mathrm{m}, 2 \mathrm{H}), 2.69(\mathrm{t}, J=5.0 \mathrm{~Hz}, 4 \mathrm{H}), 2.81-2.88(\mathrm{~m}, 2 \mathrm{H}), 3.23(\mathrm{t}, J=5.0 \mathrm{~Hz}, 4 \mathrm{H}), 6.86(\mathrm{t}, J=7.3 \mathrm{~Hz}$, $1 \mathrm{H}), 6.94(\mathrm{~d}, J=8.1 \mathrm{~Hz}, 2 \mathrm{H}), 7.17-7.33(\mathrm{~m}, 7 \mathrm{H}) ;{ }^{13} \mathrm{C}\left\{{ }^{1} \mathrm{H}\right\} \mathrm{NMR}\left(100 \mathrm{MHz}, \mathrm{CDCl}_{3}\right) \square 33.6,49.1$, 53.2, 60.5, 116.0, 119.6, 126.0, 128.4, 128.7, 129.1, 140.2, 151.2.

1-(2-Phenylethyl)-4-tert-butyl piperazinecarboxylate (Table 2, Entry 4). ${ }^{3}$ The general procedure was followed with styrene $(229 \square \mathrm{l}, 2.00 \mathrm{mmol})$ and tert-Butyl 1-piperazinecarboxylate $(187 \mathrm{mg}, 1.00 \mathrm{mmol})$. The reaction mixture was purified by flash column chromatography (20\% EtOAc in hexane) to give $263 \mathrm{mg}$ (90\%) of the hydroamination product: ${ }^{1} \mathrm{H}$ NMR (400 MHz, $\left.\mathrm{CDCl}_{3}, \mathrm{TMS}\right) \square 1.45(\mathrm{~s}, 9 \mathrm{H}), 2.46(\mathrm{t}, J=4.5 \mathrm{~Hz}, 4 \mathrm{H}), 2.56-2.64(\mathrm{~m}, 2 \mathrm{H}), 2.77-2.84(\mathrm{~m}, 2 \mathrm{H}), 3.46$ $(\mathrm{t}, J=5.0 \mathrm{~Hz}, 4 \mathrm{H}), 7.17-7.23(\mathrm{~m}, 3 \mathrm{H}), 7.25-7.32(\mathrm{~m}, 2 \mathrm{H}) ;{ }^{13} \mathrm{C}\left\{{ }^{1} \mathrm{H}\right\} \mathrm{NMR}\left(100 \mathrm{MHz}, \mathrm{CDCl}_{3}\right) \square$ 28.4, 33.4, 43.4 (broad), 52.9, 60.4, 79.5, 126.0, 128.3, 128.6, 140.0, 154.7.

$\mathrm{N}$-(2-Phenethyl)-4-piperidone ethylene ketal (Table 2, Entry 5). ${ }^{4}$ The general procedure was followed with styrene $(229 \square 1,2.00 \mathrm{mmol})$ and 4-piperidone ethylene ketal (143 $\mathrm{mg}, 1.00 \mathrm{mmol})$. The reaction mixture was purified by flash column chromatography (50\% EtOAc in hexane) to give $203 \mathrm{mg}(82 \%)$ of the hydroamination product: ${ }^{1} \mathrm{H}$ NMR (500 MHz, $\left.\mathrm{CDCl}_{3}, \mathrm{TMS}\right) \square 1.79(\mathrm{t}, J=5.7 \mathrm{~Hz}, 4 \mathrm{H}), 2.57-2.64(\mathrm{~m}, 4 \mathrm{H}), 2.61-2.67(\mathrm{~m}, 2 \mathrm{H}), 2.78-2.84(\mathrm{~m}$, $2 \mathrm{H}), 3.96(\mathrm{~s}, 4 \mathrm{H}), 7.17-7.23(\mathrm{~m}, 3 \mathrm{H}), 7.25-7.31(\mathrm{~m}, 2 \mathrm{H}) ;{ }^{13} \mathrm{C}\left\{{ }^{1} \mathrm{H}\right\}$ NMR $\left(125 \mathrm{MHz}, \mathrm{CDCl}_{3}\right) \square$ $34.2,35.1,51.6,60.4,64.5,107.5,126.2,128.6,128.9,140.8$.

$\mathrm{N}$-(2-Phenethyl)tetrahydroisoquinoline (Table 2, Entry 6). ${ }^{5}$ The general procedure was followed with styrene (458 $\square \mathrm{l}, 4.00 \mathrm{mmol})$ and 1, 2, 3, 4-tetrahydro-isoquinoline (136 mg, $1.02 \mathrm{mmol}$ ). The reaction mixture was purified by flash column chromatography (20\% EtOAc in hexane) to give $157 \mathrm{mg}(65 \%)$ of the hydroamination product: ${ }^{1} \mathrm{H}$ NMR $\left(500 \mathrm{MHz}, \mathrm{CDCl}_{3}\right.$, TMS) $\square$ 2.75-2.81 (m, 2H), $2.81(\mathrm{t}, J=5.8 \mathrm{~Hz}, 2 \mathrm{H}), 2.89-2.96(\mathrm{~m}, 4 \mathrm{H}), 3.72(\mathrm{~s}, 2 \mathrm{H}), 7.01-7.06$ (m, 1H), 7.08-7.16 (m, 3H), 7.18-7.23 (m, 1H), 7.23-7.27 (m, 2H), 7.27-7.32 (m, 2H); ${ }^{13} \mathrm{C}\left\{{ }^{1} \mathrm{H}\right\}$ NMR $\left(125 \mathrm{MHz}, \mathrm{CDCl}_{3}\right) \square 29.3,34.2,51.2,56.3,60.5,125.8,126.3,126.3,126.8,128.6,128.9$, 129.0, 134.5, 134.9, 140.6 .

$N$-(2-Phenethyl)hexylmethylamine (Table 2, Entry 7). ${ }^{6}$ The general procedure was followed with styrene (458 $\square \mathrm{l}, 4.00 \mathrm{mmol})$ and $N$-methyhexyllamine $(115 \mathrm{mg}, 1.00 \mathrm{mmol})$. The reaction mixture was stirred at $80{ }^{\circ} \mathrm{C}$ for $48 \mathrm{~h}$. After the heating, the reaction mixture was purified by flash column chromatography (20\% EtOAc in hexane) to give $138 \mathrm{mg}(63 \%)$ of the 
hydroamination product: ${ }^{1} \mathrm{H}$ NMR (500 $\mathrm{MHz}, \mathrm{CDCl}_{3}$, TMS) $\square 0.88$ (t, J=7.0 Hz, 3H), 1.24-1.35 (m, 6H), 1.42-1.52 (m, 2H), $2.30(\mathrm{~s}, 3 \mathrm{H}), 2.39$ (t, $J=7.7 \mathrm{~Hz}, 2 \mathrm{H}), 2.57-2.63$ (m, 2H), 2.74-2.80 (m, 2H), 7.16-7.22 (m, 3H), 7.25-7.30 (m, 2H); ${ }^{13} \mathrm{C}\left\{{ }^{1} \mathrm{H}\right\} \mathrm{NMR}\left(125 \mathrm{MHz}, \mathrm{CDCl}_{3}\right) \square$ 14.3, 22.9, 27.5, 27.6, 32.1, 34.1, 42.5, 58.0, 59.9, 126.1, 128.6, 128.9, 140.9 .

$N$-(2-Phenethyl]benzylmethylamine (Table 2, Entry 8). ${ }^{7}$ The general procedure was followed with styrene (458 $\square 1,4.00 \mathrm{mmol})$ and $N$-methyl benzylamine (121 $\mathrm{mg}, 1.00 \mathrm{mmol})$. The reaction mixture was stirred at $80{ }^{\circ} \mathrm{C}$ for $48 \mathrm{~h}$. After the heating, the reaction mixture was purified by flash column chromatography (10\% EtOAc in hexane) to give $113 \mathrm{mg}(50 \%)$ of the hydroamination product: ${ }^{1} \mathrm{H}$ NMR $\left(500 \mathrm{MHz}, \mathrm{CDCl}_{3}\right.$, TMS) $\square 2.28(\mathrm{t}, J=2.4 \mathrm{~Hz}, 3 \mathrm{H}), 2.62-2.67$ $(\mathrm{m}, 2 \mathrm{H}), 2.79-2.86(\mathrm{~m}, 2 \mathrm{H}), 3.56(\mathrm{~s}, 2 \mathrm{H}), 7.16-7.21(\mathrm{~m}, 3 \mathrm{H}), 7.21-7.33(\mathrm{~m}, 7 \mathrm{H}) ;{ }^{13} \mathrm{C}\left\{{ }^{1} \mathrm{H}\right\} \mathrm{NMR}$ $\left(125 \mathrm{MHz}, \mathrm{CDCl}_{3}\right) \square 34.2,42.4,59.4,62.4,126.1,127.1,128.4,128.5,129.0,129.2,139.3$, 140.8 .

$N$-[2-(2, 5-Dimethylphenyl)ethyl]morpholine (Table 2, Entry 9). The general procedure was followed with 2, 5-dimethylstyrene (293 $\square 1,2.00 \mathrm{mmol})$ and morpholine (86.9 $\mathrm{mg}, 1.00 \mathrm{mmol})$. The reaction mixture was purified by flash column chromatography (20\% EtOAc in hexane) to give $177 \mathrm{mg}(81 \%)$ of the hydroamination product. ${ }^{1} \mathrm{H}$ NMR $(500 \mathrm{MHz}$, $\left.\mathrm{CDCl}_{3}, \mathrm{TMS}\right) \square 2.30(\mathrm{~s}, 3 \mathrm{H}), 2.30(\mathrm{~s}, 3 \mathrm{H}), 2.51-2.57(\mathrm{~m}, 2 \mathrm{H}), 2.53-2.60(\mathrm{~m}, 4 \mathrm{H}), 2.75-2.81(\mathrm{~m}$, 2H), $3.77(\mathrm{t}, J=4.7 \mathrm{~Hz}, 4 \mathrm{H}), 6.94(\mathrm{~d}, J=7.7 \mathrm{~Hz}, 1 \mathrm{H}), 6.97(\mathrm{~s}, 1 \mathrm{H}), 7.04(\mathrm{~d}, J=7.7 \mathrm{~Hz}, 1 \mathrm{H})$; ${ }^{13} \mathrm{C}\left\{{ }^{1} \mathrm{H}\right\} \mathrm{NMR}\left(125 \mathrm{MHz}, \mathrm{CDCl}_{3}\right) \square 19.1,21.1,30.7,54.0,60.0,67.2,127.2,130.3,130.4,133.0$, 135.7, 138.2; Anal. Calcd for $\mathrm{C}_{14} \mathrm{H}_{21} \mathrm{NO}$ : C, 76.67; H, 9.65; N, 6.39. Found: C, 76.95; H, 9.90; N, 6.21 .

$N$-[2-(4-Methylphenyl)ethyl]morpholine (Table 2, Entry 10). ${ }^{2}$ The general procedure was followed with 4-methylstyrene (264 $\square \mathrm{l}, 2.00 \mathrm{mmol})$ and morpholine $(88.5 \mathrm{mg}, 1.02 \mathrm{mmol})$. The reaction mixture was purified by flash column chromatography (20\% EtOAc in hexane) to give $150 \mathrm{mg}(72 \%)$ of the hydroamination product. ${ }^{1} \mathrm{H}$ NMR (400 $\left.\mathrm{MHz}, \mathrm{CDCl}_{3}, \mathrm{TMS}\right) \square 2.31$ (s, $3 \mathrm{H}), 2.52(\mathrm{t}, J=4.5 \mathrm{~Hz}, 4 \mathrm{H}), 2.57(\mathrm{~m}, 2 \mathrm{H}), 2.76(\mathrm{~m}, 2 \mathrm{H}), 3.74(\mathrm{t}, J=4.5 \mathrm{~Hz}, 4 \mathrm{H}), 7.09$ (s, 4H); ${ }^{13} \mathrm{C}\left\{{ }^{1} \mathrm{H}\right\} \mathrm{NMR}\left(100 \mathrm{MHz}, \mathrm{CDCl}_{3}\right) \square 20.9,32.7,53.6,60.9,66.8,128.4,129.0,135.4,136.9$.

$N$-[2-(3-Methoxyphenyl)ethyl]morpholine (Table 2, Entry 11). The general procedure was followed with 3-methoxystyrene (278 $\square 1,2.00 \mathrm{mmol})$, morpholine (86.5 mg, $0.99 \mathrm{mmol})$ and $0.25 \mathrm{~mL}$ of 1, 4-dioxane. The reaction mixture was stirred at $110{ }^{\circ} \mathrm{C}$ for $48 \mathrm{~h}$. After the heating, the reaction mixture was purified by flash column chromatography $(20 \%$ EtOAc in 
hexane) to give $200 \mathrm{mg}(91 \%)$ of the hydroamination product. ${ }^{1} \mathrm{H} \mathrm{NMR}\left(500 \mathrm{MHz}, \mathrm{CDCl}_{3}\right.$, TMS) $\square 2.47-2.56(\mathrm{~m}, 4 \mathrm{H}), 2.57-2.63(\mathrm{~m}, 2 \mathrm{H}), 2.76-2.81(\mathrm{~m}, 2 \mathrm{H}), 3.74(\mathrm{t}, J=4.5 \mathrm{~Hz}, 4 \mathrm{H}), 3.80$ $(\mathrm{s}, 3 \mathrm{H}), 6.73-6.78(\mathrm{~m}, 1 \mathrm{H}), 6.76(\mathrm{~s}, 1 \mathrm{H}), 6.80(\mathrm{~d}, J=7.6 \mathrm{~Hz}, 1 \mathrm{H}), 7.17-7.23(\mathrm{~m}, 1 \mathrm{H}) ;{ }^{13} \mathrm{C}\left\{{ }^{1} \mathrm{H}\right\}$ NMR (125 MHz, $\left.\mathrm{CDCl}_{3}\right) \square 33.6,53.9,55.4,60.9,67.2,111.5,114.8,121.3,129.6,142.0,159.9$; Anal. Calcd for $\mathrm{C}_{13} \mathrm{H}_{19} \mathrm{NO}_{2}$ : C, 70.56; H, 8.65; N, 6.33. Found: C, 70.46; H, 8.94; N, 6.28.

$N$-[2-(3-Trifluoromethylphenyl)ethyl]morpholine (Table 2, Entry 12). ${ }^{2}$ The general procedure was followed with 3-trifluoromethylstyrene (297 $\square 1,2.00 \mathrm{mmol})$, morpholine (87.2 $\mathrm{mg}, 1.00 \mathrm{mmol}$ ) and $0.25 \mathrm{~mL}$ of 1, 4-dioxane. The reaction mixture was stirred at $110{ }^{\circ} \mathrm{C}$ for 24 h. After the heating, the reaction mixture was purified by flash column chromatography $(20 \%$ EtOAc in hexane) to give $185 \mathrm{mg}(71 \%)$ of the hydroamination product. ${ }^{1} \mathrm{H} \mathrm{NMR}(400 \mathrm{MHz}$, $\left.\mathrm{CDCl}_{3}, \mathrm{TMS}\right) \square 2.45-2.55(\mathrm{~m}, 4 \mathrm{H}), 2.58-2.65(\mathrm{~m}, 2 \mathrm{H}), 2.84-2.92(\mathrm{~m}, 2 \mathrm{H}), 3.76(\mathrm{t}, J=4.6 \mathrm{~Hz}$, 4H), 7.39-7.44 (m, 2H), 7.46-7.52 (m, 2H); ${ }^{13} \mathrm{C}\left\{{ }^{1} \mathrm{H}\right\}$ NMR (100 MHz, $\left.\mathrm{CDCl}_{3}\right) \square 33.0,53.6,60.3$, $66.9,123.0(\mathrm{q}, J=3.8 \mathrm{~Hz}), 124.2(\mathrm{q}, J=272.2 \mathrm{~Hz}), 125.4(\mathrm{q}, J=3.8 \mathrm{~Hz}), 128.8,130.7(\mathrm{q}, J=$ $32.2 \mathrm{~Hz}), 132.1,141.0$.

N-[2-(4-Trifluoromethylphenyl)ethyl]morpholine (Table 2, Entry 13). The general procedure was followed with 4-trifluoromethylstyrene (296 $\square 1,2.00 \mathrm{mmol})$ and morpholine (87.4 $\mathrm{mg}, 1.00 \mathrm{mmol}$ ). The reaction mixture was purified by flash column chromatography (40\% EtOAc in hexane) to give $131 \mathrm{mg}(51 \%)$ of the hydroamination product. ${ }^{1} \mathrm{H} \mathrm{NMR}(500 \mathrm{MHz}$, $\left.\mathrm{CDCl}_{3}, \mathrm{TMS}\right) \square 2.52(\mathrm{t}, J=4.1 \mathrm{~Hz}, 4 \mathrm{H}), 2.58-2.63(\mathrm{~m}, 2 \mathrm{H}), 2.83-2.89(\mathrm{~m}, 2 \mathrm{H}), 3.73(\mathrm{t}, J=4.7$ $\mathrm{Hz}, 4 \mathrm{H}), 7.32(\mathrm{~d}, J=7.9 \mathrm{~Hz}, 2 \mathrm{H}), 7.54(\mathrm{~d}, J=7.9 \mathrm{~Hz}, 2 \mathrm{H}) ;{ }^{13} \mathrm{C}\left\{{ }^{1} \mathrm{H}\right\} \mathrm{NMR}\left(125 \mathrm{MHz}, \mathrm{CDCl}_{3}\right) \square$ $33.4,53.9,60.5,67.2,124.5(\mathrm{q}, J=271.7 \mathrm{~Hz}), 125.5(\mathrm{q}, J=3.6 \mathrm{~Hz}), 128.7(\mathrm{q}, J=33.1 \mathrm{~Hz})$, 129.2, 144.6; Anal. Calcd for $\mathrm{C}_{13} \mathrm{H}_{16} \mathrm{~F}_{3} \mathrm{NO}$ : C, 60.22; H, 6.22; N, 5.40. Found: C, 60.05; H, 6.25; N, 5.34 .

N-1-(2-Phenylpropyl)morpholine (Table 2, Entry 14). ${ }^{8}$ The general procedure was followed with $\square$-methylstyrene (195 $\square 1,1.50 \mathrm{mmol})$ and morpholine (88.2 $\mathrm{mg}, 1.01 \mathrm{mmol})$. The reaction mixture was stirred at $80{ }^{\circ} \mathrm{C}$ for $72 \mathrm{~h}$. After the heating, the reaction mixture was purified by flash column chromatography (20\% EtOAc in hexane) to give $83 \mathrm{mg}(40 \%)$ of the hydroamination product. ${ }^{1} \mathrm{H}$ NMR (500 MHz, $\left.\mathrm{CDCl}_{3}, \mathrm{TMS}\right) \square 1.28(\mathrm{~d}, J=7.0 \mathrm{~Hz}, 3 \mathrm{H}), 2.34-2.41$ (m, 2H), 2.42-2.51 (m, 4H), $2.94(\mathrm{ddd}, J=13.9,8.4,6.9 \mathrm{~Hz}, 1 \mathrm{H}), 3.63-3.72(\mathrm{~m}, 4 \mathrm{H}), 7.17-7.23$ $(\mathrm{m}, 3 \mathrm{H}), 7.27-7.32(\mathrm{~m}, 2 \mathrm{H}) ;{ }^{13} \mathrm{C}\left\{{ }^{1} \mathrm{H}\right\} \mathrm{NMR}\left(125 \mathrm{MHz}, \mathrm{CDCl}_{3}\right) \square$ 20.1, 37.3, 54.2, 66.8, 67.3, 126.3, 127.4, 128.5, 146.2. 
Reactions of 2, 5-Dimethyl Styrene with Morpholine in the Presence of $N$-(1Phenethyl)morpholine (eq 2). In a drybox, morpholine (17.4 $\square 1,0.20 \mathrm{mmol}$ ), 2, 5-dimethyl styrene $(58.5 \square 1,0.40 \mathrm{mmol})$ and $N$-(1-phenethyl)morpholine $(38.2 \mathrm{mg}, 0.20 \mathrm{mmol})$ were added to a screw-capped vial containing a suspension of $\mathrm{Ru}(\mathrm{cod})(2 \text {-methylallyl) })_{2}(3.2 \mathrm{mg}, 10 \square \mathrm{mol})$ and 1, 5-bis(diphenylphosphino)pentane $(6.2 \mathrm{mg}, 14 \square \mathrm{mol})$ in $0.10 \mathrm{~mL}$ of 1, 4-dioxane. The vial was sealed with a cap containing a PTFE septum and removed from the drybox. Triflic acid (1.8 $\square 1,20 \square \mathrm{mol}$ ) was added by syringe to the suspension. The reaction mixture was stirred at 100 ${ }^{\circ} \mathrm{C}$. After $24 \mathrm{~h}$, the molar ratios of $N$-(2-phenethyl)morpholine, $N$-[2-(2, 5dimethylphenyl)ethyl]-morpholine and $N$-(1-phenethyl)morpholine were determined to be 0:76:99 by GC analysis after addition of dodecane as internal standard.

Reactions of 2, 5-Dimethyl Styrene with Morpholine in the Presence of $N$-(2Phenylethyl)-4-tert-butyl piperazinecarboxylate (eq 3). In a drybox, morpholine (17.4 $\square 1$, $0.20 \mathrm{mmol}), 2$, 5-dimethyl styrene (58.5 $\square 1,0.40 \mathrm{mmol})$ and 1-(2-phenethyl)-4-tert-butyl piperazinecaroxylate $(58.1 \mathrm{mg}, 0.20 \mathrm{mmol})$ were added to a screw-capped vial containing a suspension of $\mathrm{Ru}(\mathrm{cod})(2$-methylallyl) 2 (3.2 $\mathrm{mg}, 10 \square \mathrm{mol})$ and 1, 5-bis(diphenylphosphino)pentane $(6.2 \mathrm{mg}, 14 \square \mathrm{mol})$ in $0.10 \mathrm{~mL}$ of 1 , 4-diioxane. The vial was sealed with a cap containing a PTFE septum and removed from the drybox. Triflic acid (1.8 $\square$ l, $20 \square \mathrm{mol})$ was added by syringe to the suspension. The reaction mixture was stirred at $100{ }^{\circ} \mathrm{C}$. After $24 \mathrm{~h}$, the yields of $N$-[2-(2, 5-dimethylphenyl)ethyl]morpholine and $N$-(2-phenethyl)morpholine were determined as $78 \%$ and $0 \%$ by GC analysis after addition of dodecane as internal standard. The remaining amount of 1-(2-phenethyl)-4-tert-butyl piperazinecaroxylate was determined to be $93 \%$ of the original quantity by ${ }^{1} \mathrm{H}-\mathrm{NMR}$ spectroscopy with dodecane as the internal standard.

Reactions of Styrene with Piperidine in the Presence of $(E)-N-(2-$ Phenylethenyl)morpholine (eq 4). In a drybox, piperidine (19.8 $\square 1,0.20 \mathrm{mmol}$ ), styrene (45.8 $\square 1,0.40 \mathrm{mmol})$ and $(E)-N$-(2-phenylethenyl)morpholine $(3.8 \mathrm{mg}, 20 \square \mathrm{mol})$ were added to a screw-capped vial containing a suspension of $\mathrm{Ru}(\mathrm{cod})(2 \text {-methylallyl) })_{2}(6.4 \mathrm{mg}, 20 \square \mathrm{mol})$ and 1, 5-bis(diphenylphosphino)pentane (12.4 mg, $28 \square \mathrm{mol})$ in $0.10 \mathrm{~mL}$ of 1, 4-dioxane. The vial was sealed with a cap containing a PTFE septum and removed from the drybox. Triflic acid (1.8 $\square$, $20 \square \mathrm{mol}$ ) was added by syringe to the suspension. The reaction mixture was stirred at $100{ }^{\circ} \mathrm{C}$. After $24 \mathrm{~h}$, the molar ratios of $N$-(2-phenethyl)piperidine, $(E)-N$-(2-phenylethenyl)piperidine, $N$ (2-phenethyl)morpholine and (E)-N-(2-phenylethenyl)morpholine were determined to be 
94:0:5:5 by GC analysis after addition of dodecane as internal standard.

\section{References}

(1) Ernst, R. D.; Melendez, E.; Stahl, L. Organometallics 1991, 10, 3636.

(2) Beller, M.; Trauthwein, H.; Eichberger, M.; Breindl, C.; Herwig, J.; Müller, T. E.; Thiel, O. R. Chem. Eur. J. 1999, 5, 1306.

(3) Utsunomiya, M.; Kuwano, R.; Kawatsura, M.; Hartwig, J. F. J. Am. Chem. Soc. 2003, 125,5608 .

(4) Dutta, A. K.; Xu, C.; Reith, M. E. A. J. Med. Chem. 1996, 39, 749.

(5) Maeda, D. Y.; Williams, W.; Kim, W. E.; Thatcher, L. N.; Bowen, W. D.; Coop, A. Bioorg. Med. Chem. Lett. 2002, 12, 497.

(6) Blaney, P.; Grigg, R.; Rankovic, Z.; Thoroughgood, M. Tetrahedron Lett. 2000, 41, 6635 .

(7) Lepley, A. R.; Giumanini, A. G. J. Org. Chem. 1967, 32, 1706.

(8) Rische, T.; Eilbracht, P. Synthesis 1997, 1331. 